\title{
Rare cause of unexplained hypertension in an oligomenorrheic adolescent girl with adnexal mass: case report and review of literature
}

\author{
Ankita Sethi $^{1}$, Vidushi Kulshrestha ${ }^{1 *}$, Seema Kaushal ${ }^{2}$, Neerja Bhatla ${ }^{1}$ \\ ${ }^{1}$ Department of Obstetrics and Gynaecology, ${ }^{2}$ Department of Pathology, AIIMS, New Delhi, India \\ Received: 17 August 2021 \\ Accepted: 07 September 2021 \\ *Correspondence: \\ Dr. Vidushi Kulshrestha, \\ E-mail: drvidushi.kul@gmail.com \\ Copyright: $\odot$ the author(s), publisher and licensee Medip Academy. This is an open-access article distributed under \\ the terms of the Creative Commons Attribution Non-Commercial License, which permits unrestricted non-commercial \\ use, distribution, and reproduction in any medium, provided the original work is properly cited.
}

\begin{abstract}
A 17-year-old girl with oligomenorrhoea was detected to have hypertension and right adnexal solid-cystic mass, $6 \times 8$ $\mathrm{cm}$ on ultrasound. Hormone profile was normal, CA-125 was $132.5 \mathrm{U} / \mathrm{mL}$, other tumour markers were in normal range; though RMI-4 and ROMA index suggested malignancy. Patient underwent exploratory laparotomy, peritoneal wash cytology, right salpingo-oophorectomy, pelvic lymphadenectomy and omental biopsy. Intraoperative frozen-section revealed ovarian steroid cell tumour. Later, steroid cell tumour-not otherwise specified (SCT-NOS) was confirmed on histopathology and immunohistochemistry. When followed three months post-surgery, patient had resumed 30-32 days menstrual-cycle, and she was normotensive without medications. This case is being reported to emphasize that imaging though not recommended for evaluation of PCOS in adolescence; and measuring blood-pressure which is often skipped in young women; proved crucial in this patient.
\end{abstract}

Keywords: Adolescent, Ovarian mass, Hypertension, Steroid cell tumour

\section{INTRODUCTION}

Oligomenorrhea in adolescents is not uncommon especially within two years of menarche before hypothalmo-pituitary-ovarian axis maturation and is also a common clinical manifestation of polycystic ovarian syndrome (PCOS) which commonly affects the adolescents. More often than not, ultrasound is skipped while evaluating suspected PCOS, it being only an optional investigation in the adolescents. ${ }^{1}$ However, imaging may unravel an unsuspected cause.

\section{CASE REPORT}

A 17-year-old girl with oligomenorrhoea for one year, was clinically diagnosed as polycystic ovarian syndrome (PCOS) and prescribed combined oral contraceptive pills (OCPs), on which her menstrual cycles became regular. Three months after stopping OCPs, she again was oligomenorhoeic and presented to our institute for a second opinion. Patient had attained menarche at 13 years and had regular menses till one year back. She had no hyperandrogenic symptoms and no family history of cancer.

On examination, patient was overweight (BMI 25.3 $\mathrm{kg} / \mathrm{m}^{2}$ ), blood pressure was $150 / 90 \mathrm{~mm} \mathrm{Hg}$, the modified Ferriman Gallaway score was four and breast was Tanner stage 4. Hormone profile was normal (serum LH 1.10 $\mathrm{mIU} / \mathrm{ml}$, serum FSH $1.61 \mathrm{mIU} / \mathrm{ml}$, Prolactin $13.52 \mathrm{ng} / \mathrm{ml}$, TSH $2.23 \mu \mathrm{IU} / \mathrm{ml}$, DHEAS <15 ng/ml, Total Testosterone $26.20 \mathrm{ng} / \mathrm{ml}$ ). Ultrasound revealed a solid-cystic right adnexal mass with predominant solid component. Contrast-enhanced computed tomography confirmed the same $8 \times 5 \times 5 \mathrm{~cm}$ solid-cystic mass with clear fat planes with surrounding structures. Serum CA-125 was $132.5 \mathrm{U} / \mathrm{mL}$, serum AFP $7.22 \mathrm{ng} / \mathrm{mL}$, beta HCG $<2.39 \mathrm{mIU} / \mathrm{mL}$ and HE4 $66.7 \mathrm{pg} / \mathrm{mL}$. RMI-4 was $528(2 \times 2 \times 132 \times 1)$ and 
ROMA index was 15.4, which was suggestive of malignancy. During the preoperative workup, the patient was diagnosed with unexplained essential hypertension and antihypertensives were started.

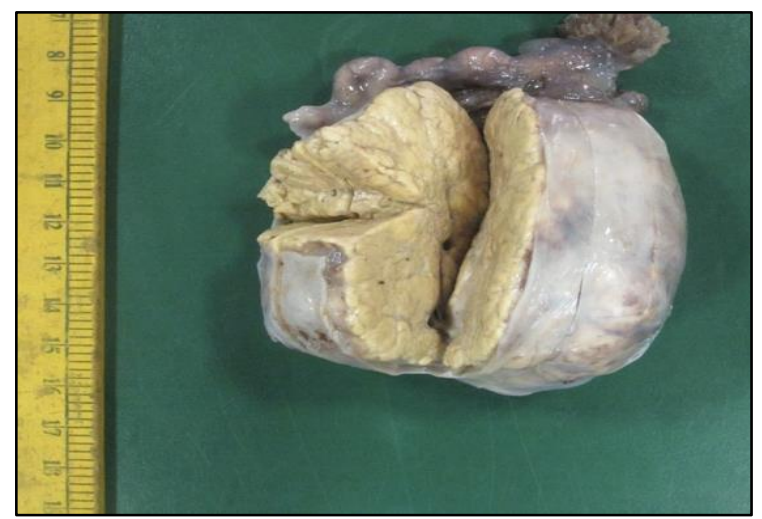

Figure 1: Gross specimen showing completely solid tumour with a yellowish surface without capsular breach.

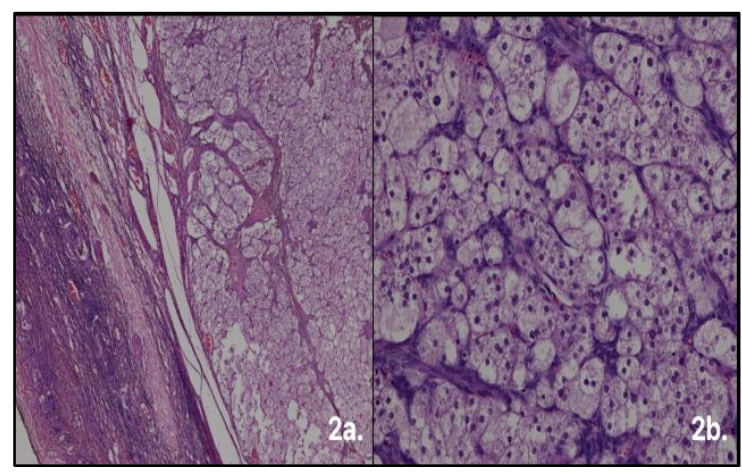

Figure 2: Histopathological examination (a) at 20X, (b) at 40X magnification showing tumour cells arranged in sheets separated by bands. Tumour cells are round to polygonal with pale cytoplasm and round nuclei.

The patient underwent exploratory laparotomy. On systematic exploration, liver, under-surface of the diaphragm, stomach, large intestine, small intestine, paracolic gutters, mesentery, pelvic peritoneum, uterus, left tube and ovary were normal with no enlarged lymph nodes. There was a right adnexal mass $8 \times 6 \mathrm{~cm}$ with no surface deposits or capsular breach or papillary projections. Peritoneal wash cytology was taken, and right salpingo-oophorectomy was done, with an intent of preserving fertility. Intraoperative frozen section analysis revealed ovarian steroid cell tumour. Right pelvic lymphadenectomy and omental biopsy were done for staging and assessing the need for adjuvant therapy, if any.

Cut section of right ovary showed a completely yellow solid tumour and no capsular breach (Figure 1). Peritoneal wash cytology had no malignant cells. Histopathology showed tumour cells arranged in sheets separated by bands. Tumour cells were round to polygonal with pale cytoplasm and round nuclei. No mitosis, necrosis, or cellular atypia was noted, and the fallopian tube was histologically unremarkable. Tumour cells were immunepositive for inhibin and calretinin, negative for cytokeratin and vimentin. MIB 1 (cell proliferation marker) labelling index was $<1 \%$. Overall features were compatible with Steroid cell tumour-Not otherwise specified (SCT-NOS) (Figure 2). No adjuvant therapy was required.

On follow-up at three months post-surgery, patient had resumed her 30-32 days menstrual cycle, and blood pressure was within normal limits. She remained normotensive over the next one year of follow-up, indicating that the etio-pathogenesis of hypertension was likely due to the pro-hypertensive hormones produced by the ovarian neoplasm.

\section{DISCUSSION}

The index case was an adolescent girl with oligomenorrhea, unilateral adnexal mass and unexplained hypertension with final diagnosis of SCT-NOS on histopathology, which is amongst the rarest ovarian tumours. This case is being reported to emphasize that imaging though not recommended for evaluating an adolescent female with features of polycystic ovarian syndrome; proved crucial in this patient and led to her optimum management. This is also to highlight that general physical examination can add to vital clinical information. Only one case of SCT presenting with adnexal mass and refractory hypertension is reported till date. ${ }^{2}$ Here we present another case along with a brief review.

SCTs are sex cord-stromal tumours and constitute less than 0.1 percent of all ovarian tumours. ${ }^{3} \mathrm{SCT}$, not otherwise specified (SCT-NOS), accounts for $60 \%$ of SCTs and are usually seen in young women. ${ }^{4}$ These tumours may be associated with hyperandrogenic or hyperestrogenic changes and may also secrete adrenal steroid hormones causing hypercortisolism. ${ }^{5}$

SCTs are hard to diagnose, and require evaluation of clinical, histological, and immunohistochemistry (IHC) findings for correct diagnosis. These tumours can appear at any age but are rare before puberty, with a mean age of 43 years and most are unilateral, only $6 \%$ being bilateral. ${ }^{6}$ The majority of patients affected with SCT-NOS show symptoms of hyperandrogenism like virilization, hirsutism, clitoromegaly, acne, change in voice, and baldness. About 10-20\% will show hyperestrogenism which manifests as postmenopausal bleeding, menorrhagia, and, in rare cases, even endometrial carcinoma. ${ }^{6}$ Around $6-10 \%$ are clinically associated with hypercortisolism and present with symptoms like hypertension, while $25 \%$ of SCT-NOS are nonfunctioning. ${ }^{7}$

This patient had only oligo-amenorrhoea with no hyperandrogenic or hyperestrogenic symptom and normal 
hormone profile unlike most of the reported cases who had hyperandrogenic features and raised testosterone levels. Another feature in this patient was hypertension which resolved spontaneously after surgery, showing a correlation between ovarian tumour and hypertension, similar to the case reported by Lee et al. ${ }^{2}$ SCT can induce hypertension. One of the hypotheses for this is that these cells become neoplastic, causing previously silenced genes to become functional, resulting in the eventual activation of enzymes such as RAS (renin-angiotensin-system) pathway in the adrenocortical tissue. ${ }^{8}$ The ovarian RAS pathway plays a crucial role in reproductive function, including folliculogenesis, oocyte maturation, ovulation, steroid hormone synthesis and corpus luteum formation. ${ }^{2}$ Normally, ovaries contain a significantly small volume of secreted renin, so hypertension attributable to ovaryderived renin is seen only in the scenario of ovarian tumours. Owing to co-existing hypertension, it appears that SCT in this patient, was either a renin or cortisol producing type; with cortisol producing SCT being less likely as there was no other feature of hypercortisolism except hypertension. Spontaneous control of hypertension after removing the tumour points to the possibility of renin producing ovarian SCT. Till patient's last follow-up one year post-surgery, patient did not develop hypertension again nor were there any signs of recurrence on clinical and radiological evaluation.

SCT is characterized as a tumour made up almost entirely of steroid-like cells deficient in Reinke crystals. ${ }^{3}$ SCT is well-circumscribed solid tumour, often lobulated, with an average diameter of $8.4 \mathrm{~cm}$. They are brown, red, or rarely black; haemorrhage within the tumour mass is uncommon. ${ }^{3}$ In our case, the cut section showed a completely solid tumour with yellowish surface and no cystic degeneration or haemorrhage. There was no mitosis, necrosis or cellular atypia, indicating its benign nature.

SCTs are immunohistochemically positive for steroidogenic factor-1, calretinin, and inhibin. They are generally negative for FOXL2 and positive for Melan A. ${ }^{3}$ Inhibin is the most useful of all these markers since most SCTs express this marker. In our case, tumour cells were immuno-positive for inhibin and calretinin, negative for cytokeratin, vimentin and MIB 1 labelling index was <1 percent.

The first-line management of SCT is optimal debulking. In stage IA disease affecting young females, fertility preservation is feasible with only unilateral salpingooophorectomy, unilateral pelvic lymphadenectomy and omental biopsy; as was done in our patient. Malignant SCT-NOS should be treated with total abdominal hysterectomy, bilateral salpingo-oophorectomy, pelvic lymphadenectomy and omental sampling supplemented; with adjuvant chemotherapy based on the histological appearance and surgical staging. Data are limited regarding the efficacy of chemotherapy or radiotherapy for SCTs. There is no proven chemotherapy regime, but bleomycin, etoposide and cisplatin (BEP) are commonly used. ${ }^{9}$ GnRH agonists have been used as a treatment for recurrent SCT because they have an inhibitory influence on ovarian steroid hormone production. ${ }^{9}$ Recent publications on incidence of lymph node metastases in SCT suggest that lymphadenectomy may not be needed in these patients. ${ }^{10}$

\section{CONCLUSION}

Features of PCOS with complex solid-cystic adnexal mass with unexplained hypertension should raise a suspicion of SCT. The index case is unusual because SCT-NOS is amongst the rarest ovarian tumours and co-existing hypertension suggesting renin producing SCTs are even rarer. Besides, this case emphasizes the role of ultrasound even in seemingly innocuous clinically diagnosed PCOS cases in adolescents where per-vaginal examination is usually avoided and imaging is not considered essential. Imaging proved crucial in this patient and led to her optimum management.

\section{ACKNOWLEDGEMENTS}

Authors would like to acknowledge the department of Obstetrics and Gynaecology, AIIMS, New Delhi for infrastructural and intellectual support.

\section{Funding: No funding sources \\ Conflict of interest: None declared \\ Ethical approval: Not required}

\section{REFERENCES}

1. Trent M, Gordon CM. Diagnosis and Management of Polycystic Ovary Syndrome in Adolescents. Pediatrics. 2020;145(2):S210-8.

2. Lee SH, Kang MS, Lee GS, Chung WY. Refractory Hypertension and Isosexual Pseudoprecocious Puberty Associated with Renin-Secreting Ovarian Steroid Cell Tumor in a Girl. J Korean Med Sci. 2011;26(6):836.

3. Liu A, Sun J, Shao W, Jin H, Song W. Steroid cell tumors, not otherwise specified (NOS), in an accessory ovary: a case report and literature review. Gynec Oncol. 2005;97(1):260-2.

4. Kurman RJ. International Agency for Research on Cancer, World Health Organization, editors. WHO classification of tumours of female reproductive organs. 4th ed. Lyon. International Agency for Research on Cancer. 2014;307.

5. Chen VW, Ruiz B, Killeen JL, Cot TR, Wu XC, Correa CN, et al. Pathology and classification of ovarian tumors. Cancer. 2003;97(S10):2631-42.

6. Qian L, Shen Z, Zhang X, Wu D, Zhou Y. Ovarian steroid cell tumor, not otherwise specified: A case report and literature review. Molecular and Clinical Oncology. 2016;5(6):839-41.

7. Mizoguchi M, Minami S, Yamamoto M, Tanizaki Y, Kobayashi A, Ino K. Ovarian steroid cell tumor, not otherwise specified, producing testosterone: Ovarian 
steroid cell tumor, NOS. J Obstet Gynaecol Res. 2014;40(9):2081-5.

8. Faten H, Dorra G, Slim C, Wajdi S, Nadia C, Kais C, et al. Ovarian Steroid Cell Tumor (Not Otherwise Specified): A Case Report of Ovarian Hyperandrogenism. Case Reports in Oncological Medicine. 2020;2020:1-6.

9. Kim JS, Park SN, Kim BR. Recurrent ovarian steroid cell tumor, not otherwise specified managed with debulking surgery, radiofrequency ablation, and adjuvant chemotherapy. Obstet Gynecol Sci. 2014;57(6):534.

10. Nasioudis D, Kanninen TT, Holcomb K, Sisti G, Witkin SS. Prevalence of lymph node metastasis and prognostic significance of lymphadenectomy in apparent early-stage malignant ovarian sex cordstromal tumors. Gynec Oncol. 2017;145(2):243-7.

Cite this article as: Sethi A, Kulshrestha V, Kaushal $\mathrm{S}$, Bhatla N. Rare cause of unexplained hypertension in an oligomenorrheic adolescent girl with adnexal mass: case report and review of literature. Int J Reprod Contracept Obstet Gynecol 2021;10:3995-8. 